\title{
Enfermedad neumoccócica invasora en recién nacidos, antes y después de la vacunación universal con vacuna conjugada 7 y 13 valente en Uruguay
}

\author{
Elizabeth Assandri, Belén Amorín, Juan P. Gesuele, Gabriela Algorta y María Catalina Pírez
}

\section{Pneumococcal invasive disease in newborns before and after 7-valent and 13-valent universal pneumococcal vaccination in Uruguay}

Introduction: Streptococcus pneumoniae infections are not frequent in neonates, but presents high morbidity and mortality. In 2008, the 7-valent pneumococcal conjugate vaccine (PCV) was introduced in the childhood vaccination schedule and then replaced by 13 -valent PCV in 2010. First dose is given at 2 months of age. Protection of neonates is expected with universal vaccination. Objective: To describe the clinical presentation, microbiology and outcome of neonates with pneumococcal invasive infections (PII) detected in two hospitals in Uruguay in 2001-2007 (pre-vaccination), 2008 (intervention) and 2009-2013 (post-vaccination). Methods: A descriptive, retrospective study was done at Pereira Rossell Hospital and Paysandú Hospital. All isolates of S. pneumoniae obtained from normally sterile fluids were included. Data were obtained from the clinical records and the microbiology laboratory. A statistical analysis with absolute frequencies, relative, rates and relative risk was performed. Results: 25 neonates were enrolled with diagnosis of: sepsis $(n=13)$, meningitis $(n=9)$, bacteremia $(n=1)$, pneumonia with empyema $(n=1)$ and pneumonia $(n=1)$. The incidence of PII in the prevaccination period was $19 / 25$, with a rate of $0.30 / 1,000$ births, compared to post-vaccination rate of 0.04/1,000. The relative risk was 5.9. 6/20 (30\%) cases of death were reported (meningitis $n=3$; sepsis $n=2$; empyema $n=1$ ). Most common serotypes were 5 and $1(14 / 25)$ and $24 / 25$ strains were susceptible to penicillin. Discussion: The symptoms were indistinguishable to infections caused by other pathogens. PII cases decreased and no deaths occurred in the post-vaccination period. No increase in non-vaccine serotypes was observed.

Key words: Streptococcus pneumoniae, neonatal infection, conjugate neumococcal vaccine, herd immunity.

Palabras clave: Streptococcus pneumoniae, infección neonatal, vacuna neumocóccica conjugada, inmunidad de rebaño.

\section{Introducción}

$S$ treptococcus pneumoniae es uno de los principales agentes causantes de morbi-mortalidad en el mundo, principalmente en los niños bajo dos años de $\operatorname{edad}^{1,2}$. Este agente produce, en forma poco frecuente, infecciones en recién nacidos ( $\mathrm{RN})$, pero se asocian con elevada morbi-mortalidad ${ }^{2-6}$. Si bien la incidencia de estas infecciones en RN es menor a la causada por Streptococcus agalactiae, la letalidad es similar ${ }^{2}$. S. pneumoniae es causante de otitis, bacteriemia, meningitis, neumonía, sepsis, abscesos e infecciones osteo-articulares ${ }^{3,5,7}$.

En varios países la implementación y el uso de vacunas conjugadas neumocóccicas (VCN), ha determinado un impacto muy importante en la epidemiología de las infecciones graves por $S$. pneumoniae, disminuyendo drásticamente las tasas de las mismas, fundamentalmente en niños bajo dos años de edad ${ }^{5,8-14}$. Estas vacunas también han demostrado tener un efecto de inmunidad de rebaño muy importante, reduciendo la incidencia de enfermedad neumocóccica invasora (ENI) en poblaciones que no la recibieron, como los niños sobre 5 años y adultos de todas las edades ${ }^{5,9,10,15-18}$. Por cada niño vacunado, 2,2 personas no vacunadas se benefician por el efecto rebaño, atribuible, en parte, a la disminución en la colonización oro-faringea en los vacunados ${ }^{14}$. Para obtener este efecto, la vacunación debe ser universal, con una cobertura de al menos 80 a $90 \%$ de la población objetivo. Sin embargo, la vacunación no comienza hasta los 2 meses de vida, quedando excluídos los RN, los cuales tienen mayor morbi-mortalidad en el caso de adquirir enfermedades neumocóccicas ${ }^{19}$.

En Uruguay, en marzo de 2008 se incorporó en forma obligatoria y gratuita la VCN 7 valente (VCN7) en el calendario de inmunizaciones infantil, con la administración de tres dosis, a los 2, 4 y 12 meses de edad ${ }^{20}$. En febrero de 2010 la Food and Drug Administration (FDA) de Estados Unidos de América (E.U.A.) licenció una vacuna 13 valente (VCN13) y en marzo del mismo año las autoridades sanitarias de Uruguay decidieron sustituir
Facultad de Medicina. Universidad de la República Oriental del Uruguay (UdelaR). Centro Hospitalario Pereira Rossell (EA, JPG, GA, MCP). Hospital Escuela del Litoral de Paysandú (BA).

Establecimientos donde se realizó el trabajo: Centro Hospitalario Pereira Rossell y Hospital Escuela del Litoral de Paysandú.

Los autores de este artículo declaran que no existe conflicto de intereses. No existió fuente de financiamiento para la realización de la presente investigación.

Recibido: 5 de junio de 2014 Aceptado: 26 de diciembre de 2014

Correspondencia a: Elizabeth Assandri Dávila elizassandri@hotmail.com 
la anterior por esta nueva presentación ${ }^{21}$. Esta segunda vacuna contiene, además de los antígenos capsulares de VCN7 (4, 6B, 9V, 14, 18C, 19F, 23F), seis serotipos adicionales $(1,3,5,6 \mathrm{~A}, 7 \mathrm{~F}$ y 19A). Ese mismo año se ofreció una dosis de nivelación de dicha vacuna, a los niños bajo 5 años de edad ${ }^{22}$. Se alcanzaron coberturas sobre $93 \%$ en los años 2008-2009 y superiores a 95\% desde entonces. Esta cobertura vacunal sostenida mayor de $90 \%$ debería favorecer, por el efecto rebaño, la protección de individuos no vacunados ${ }^{23}$.

Está documentado el impacto que ha generado la vacunación anti-neumocóccica en la incidencia de neumonía adquirida en la comunidad y meningitis en niños bajo dos años en Uruguay, con una reducción cercana a 50\% en los casos de neumonía y de $60 \%$ en meningitis, en el período post-vacunal ${ }^{17,24}$. Es de esperar que la estrategia de vacunación implementada, tenga impacto también en la protección de los $\mathrm{RN}^{2,5}$. Si bien $S$. pneumoniae es poco frecuente como causante de infecciones en los RN, la gravedad de las mismas y el hecho de la implementación de una nueva estrategia preventiva nacional, como es la vacunación universal anti-neumocóccica, motivó a los investigadores a la realización de este estudio.

La presente investigación tuvo como objetivo describir la presentación clínica, los hallazgos microbiológicos y evolución de los RN con ENI, identificados en dos hospitales universitarios, desde el 1/1/2001 al 31/12/2007 (pre-vacunación universal anti-neumocóccica), 2008 (año de la intervención) y desde el 1/1/2009 al 31/12/2013 (post-vacunación).

\section{Material y Métodos}

Estudio descriptivo, retrospectivo, realizado en dos hospitales universitarios de Uruguay: el Centro Hospitalario Pereira Rossell (CHPR) -hospital de la mujer y pediátrico-, centro de referencia nacional, ubicado en la capital del país (Montevideo) y el Hospital Escuela del Litoral de Paysandú (HEL-P). Ambos centros hospitalarios asisten a niños de la Administración de los Servicios de Salud del Estado. En el primero de ellos se producen cerca de 8.000 nacimientos por año, que representan más de $30 \%$ de los nacimientos del departamento de Montevideo (capital del país, con una población de 1.319.108 habitantes). En el segundo centro hospitalario se producen aproximadamente 1.500 nacimientos anuales, que constituyen más de $70 \%$ de los nacimientos de ese departamento (con una población de 113.107 habitantes $)^{25,26}$.

Se identificaron todos los aislados de $S$. pneumoniae provenientes de sitios y/o líquidos normalmente estériles (sangre, líquido céfalo-raquídeo (LCR), líquido pleural, articular o hueso) de niños de entre 0 y 28 días de vida, asistidos en los dos hospitales antes mencionados. A partir de los aislados se buscaron datos de las historias clínicas de los RN y/o la historia obstétrica materna, y de los registros de la División Epidemiología del Ministerio de Salud Pública (MSP) en los casos de meningitis. De acuerdo al diseño de la investigación, se definió la población de estudio a partir de los hallazgos del laboratorio; se dispuso de los datos microbiológicos de todos los $\mathrm{RN}$ con aislamiento de $S$. pneumoniae en sitos normalmente estériles, durante los 13 años del estudio. El largo período abarcado dificultó la obtención de las historias clínicas, principalmente de los niños nacidos en el año 2001.

Los cultivos de las muestras clínicas, la identificación bacteriana y los tests de susceptibilidad a antimicrobianos se realizaron en el laboratorio de microbiología de los dos hospitales intervinientes en este estudio, siguiendo las técnicas descritas en el Manual de Microbiología Clínica de la Sociedad Americana de Microbiología ${ }^{27}$. Se consideraron susceptibles a penicilina las cepas con CIM $\leq 0,06 \mu \mathrm{g} / \mathrm{ml}$ y resistentes aquellas con CIM $\geq 0,12 \mu \mathrm{g} /$ $\mathrm{ml}$. Se consideraron susceptibles a cefotaxima las cepas con $\mathrm{CIM} \leq 0,5 \mu \mathrm{g} / \mathrm{ml}$, con susceptibilidad intermedia las cepas con CIM de $1,0 \mu \mathrm{g} / \mathrm{ml}$ y resistentes aquellas con $\mathrm{CIM} \geq 2,0 \mu \mathrm{g} / \mathrm{ml}$. Se tomaron puntos de corte para meningitis en todos los casos, dadas las características de las enfermedades invasoras en los $\mathrm{RN}^{28}$. Los aislados de $S$. pneumoniae fueron serotipificados en el laboratorio de referencia nacional (Camou T, García Gabarrot G. Servicio Nacional de Laboratorios de Salud Pública. MSP. Montevideo. Uruguay).

Se definió RN con ENI como aquel en el que se aisló $S$. pneumoniae de sitios y/o líquidos normalmente estériles (sangre, LCR, líquido pleural, articular o hueso). Se definió sepsis neonatal neumocóccica la enfermedad que presentaba un $\mathrm{RN}$ con síntomas y signos de infección sistémica en el que se aisló S. pneumoniae en sangre. La sepsis neonatal se definió como precoz si los síntomas se iniciaron dentro de los tres primeros días de vida y tardía si se iniciaron a partir del cuarto hasta el $28^{\circ}$ día de vida. Se definió como bacteriemia neumocóccica, la que presentaba un RN sin síntomas ni signos de infección, en el que se aisló $S$. pneumoniae en sangre. Se definió neumonía y/o empiema pleural neumocócico, a la entidad que presentaban aquellos $\mathrm{RN}$ con síntomas y/o signos respiratorios y radiografía de tórax compatible con neumonía y/o efusión pleural en los que se aisló $S$. pneumoniae de sangre y/o pleura. Se definió como casos de meningitis neumocóccica, aquellos $\mathrm{RN}$ en los que se aisló $S$. pneumoniae en el LCR.

Las variables analizadas fueron: edad del $\mathrm{RN}$ en el momento de aislamiento de $S$. pneumoniae, antecedentes perinatales (edad gestacional, vía del parto, tiempo de rotura de membranas, peso, test de Apgar), entidad clínica, tratamiento realizado y evolución, antecedentes de infección materna periparto, tratamiento antimicrobiano 
previo al parto, sitio de aislamiento de $S$. pneumoniae, serotipos y susceptibilidad antimicrobiana.

Se comparó el número de casos, serotipos y susceptibilidad de $S$. pneumoniae en los distintos períodos del estudio; 2001 a 2007, previo a la vacunación universal con vacuna conjugada antineumocóccica; año 2008 (año de la intervención) y 2009-2013 (post-vacunación).

El análisis de los datos se realizó en base a la distribución de frecuencias absolutas y relativas de las variables consideradas y a medidas de resumen (modo, promedio y mediana) con sus correspondientes medidas de dispersión.

Consideraciones éticas: los datos que se describen pertenecen a la vigilancia sistemática que se realiza en el CHPR y en el HEL-P, de las enfermedades neumocóccicas. Los autores son miembros de los grupos encargados de la vigilancia clínica y microbiológica.

\section{Resultados}

Durante los trece años de estudio (2001-2013) se identificaron 25 casos de ENI en RN. Veinte RN fueron hospitalizados en el CHPR y cinco en el HEL-P. Diecinueve casos ocurrieron en el período 2001-2007, representando una tasa de 0,30 por 1.000 nacimientos (IC 0,16-0,42), cuatro en el 2008, tasa de 0,46/1.000 (IC $0,00-0,92)$ y dos entre los años 2009 a 2013, tasa de 0,04 (IC 0,01-0,11). En el año 2001 ocurrieron siete casos, (tasa de 0,68/1.000 nacimientos, IC 0,17-1,20) siendo el año con mayor número. El último caso registrado fue en el año 2010. El riesgo relativo de adquisición de ENI en el período pre-vacunal comparado con el post-vacunal es de 5,98, con un valor de $\mathrm{p}=0,006$.

En la Tabla 1 se describe el diagnóstico realizado en los $\mathrm{RN}$, el sitio de aislamiento y serotipo de $S$. pneumoniae.

\section{Características de los $R \boldsymbol{N}$}

Los diagnósticos, por orden decreciente de frecuencia, fueron: sepsis (n: 13) meningitis (n: 9), bacteriemia sin identificación de foco primario (n: 1), neumonia con empiema pleural y sepsis (n: 1) y neumonía (n: 1) (Tabla 2).

Se obtuvo el dato de las horas (h) de vida al momento de obtención de la muestra bacteriológica en 21 de los 25 casos. El rango de edad fue de 0 a 28 días, con una media de 10 días, mediana de 3 y modo de menos de un día. Presentaron síntomas (fiebre, irritabilidad, síndrome de dificultad respiratoria (SDR) y apneas) después de las $72 \mathrm{~h}$ de vida, 10/21 de los RN. El rango de edad de estos $10 \mathrm{RN}$ fue de 6 a 28 días, con una media de 19 días, mediana y modo de 18 días.

En cuanto a la vía de nacimiento, se obtuvo el dato en $16 \mathrm{RN}$, siendo en 12 vía vaginal y cesárea en 4 , parto de término en 13 y de pretérmino en 3 (29, 30 y 35 semanas). Ninguno de los RN de pretérmino falleció. Sólo uno de
Tabla 1. Distribución anual de enfermedad invasora por $S$. pneumoniae en RN según diagnóstico, sitio de aislamiento y serotipo. CHPR y HEL-P. Uruguay. Años 2001 a 2013

\begin{tabular}{|c|c|c|c|c|}
\hline Caso & Año & Diagnóstico & $\begin{array}{l}\text { Sitio de aislamiento } \\
\text { de } S \text {. pneumoniae }\end{array}$ & Serotipo \\
\hline 1 & 2001 & MBA & LCR & $5^{* *}$ \\
\hline 2 & 2001 & MBA & LCR & $5^{* *}$ \\
\hline 3 & 2001 & Sepsis & Sangre & $19 A^{* *}$ \\
\hline 4 & 2001 & Sepsis & Sangre & $5^{* *}$ \\
\hline 5 & 2001 & Sepsis & Sangre & $5^{* *}$ \\
\hline 6 & 2001 & Sepsis & Sangre & $5^{* *}$ \\
\hline 7 & 2001 & MBA & LCR y sangre & 1 ** \\
\hline 8 & 2002 & Sepsis & Sangre & $11 \mathrm{~A}$ \\
\hline 9 & 2003 & Sepsis & Sangre & Pool D \\
\hline 10 & 2003 & Sepsis & Sangre & $18 C^{*}$ \\
\hline 11 & 2004 & MBA & LCR & $5^{* *}$ \\
\hline 12 & 2004 & Bacteriemia & Sangre & 1 ** \\
\hline 13 & 2005 & Sepsis & Sangre & $7 \mathrm{~F}^{* *}$ \\
\hline 14 & 2005 & MBA & LCR y sangre & 1 ** \\
\hline 15 & 2006 & MBA & LCR & 1 ** \\
\hline 16 & 2006 & Sepsis & Sangre & $18 C^{*}$ \\
\hline 17 & 2006 & MBA & LCR y sangre & $5^{* *}$ \\
\hline 18 & 2006 & MBA & LCR y sangre & $5^{* *}$ \\
\hline 19 & 2007 & Neumonía & Sangre & $19 \mathrm{~F}^{*}$ \\
\hline 20 & 2008 & MBA & LCR y sangre & $5^{* *}$ \\
\hline 21 & 2008 & Sepsis & Sangre & 8 \\
\hline 22 & 2008 & Sepsis & Sangre & No tipificado \\
\hline 23 & 2008 & Empiema/Sepsis & Sangre y líquido pleural & No tipificado \\
\hline 24 & 2009 & Sepsis & Sangre & No tipificado \\
\hline 25 & 2010 & Sepsis & Sangre & $1^{* *}$ \\
\hline
\end{tabular}

MBA: meningoencefalitis bacteriana aguda; LCR: líquido céfalo-raquídeo. *Serotipos incluidos en VCN $7 \mathrm{v}$; ${ }^{* *}$ Serotipos adicionales en VCN $13 \mathrm{v}$. Todas las cepas fueron sensibles a penicilina y cefotaxima a excepción del caso 24.

los $16 \mathrm{RN}$ presentó depresión neonatal a los 5 min de vida, apgar 3/8 (caso 9); se trató de un RN de pretérmino severo, 30 semanas, peso de nacimiento 1.665; en el que se aisló en sangre $S$. pneumoniae y $H$. influenzae, cursando asintomático y con excelente evolución.

De estos $16 \mathrm{RN}$, dos fueron pequeños para su edad gestacional (PEG) (2.200 g para 38 semanas y $2.100 \mathrm{~g}$ para 37 semanas), pero cercano al percentil 10; ambos nacimientos fueron a término y los dos pacientes fallecieron.

Ninguno de los 16 recién nacidos presentó rotura de membranas previo al parto mayor a $12 \mathrm{~h}$ y ninguno presentaba enfermedades asociadas. 
Tabla 2. Enfermedad invasora por S. pneumoniae en RN. Características clínicas. CHPR y HEL-P. Uruguay. Años 2001 al 2013

\begin{tabular}{|c|c|c|c|c|c|}
\hline Caso & Año & Edad & Diagnóstico & Otras características & Evolución \\
\hline 1 & 2001 & 28 días & MBA & S. pneumoniae aislado en $\mathrm{LCR}$, sin datos clínicos & Fallece \\
\hline 2 & 2001 & 24 días & MBA & S. pneumoniae aislado en $\mathrm{LCR}$, sin datos clínicos & Fallece \\
\hline 3 & 2001 & & Sepsis & S. pneumoniae aislado en sangre, sin datos clínicos & \\
\hline 4 & 2001 & & Sepsis & S. pneumoniae aislado en sangre, sin datos clínicos & \\
\hline 5 & 2001 & & Sepsis & S. pneumoniae aislado en sangre, sin datos clínicos & \\
\hline 6 & 2001 & & Sepsis & S. pneumoniae aislado en sangre, sin datos clínicos & \\
\hline 7 & 2001 & 6 días & MBA & $\begin{array}{l}\text { Parto: } 40 \text { sem. REM } 1 \text { h. PN } 3.650 \mathrm{~g} \text {. Apgar 9/10. No ATB previo. A los } 6 \text { días fiebre + irritabilidad. } \\
\text { S. pneumoniae aislado en LCR y sangre. Recibe ampicilina y cefotaxima. Sepsis materna }\end{array}$ & $\begin{array}{l}\text { Alta. Secuela: hipotonía } \\
\text { y convulsiones }\end{array}$ \\
\hline 8 & 2002 & $<1$ día & Sepsis & $\begin{array}{l}\text { Parto: } 40 \text { sem. PN } 3.730 \mathrm{~g} \text { Apgar 6/8. RM } 12 \text { h. No ATB previo a parto. SDR. S. pneumoniae aislado en } \\
\text { sangre. Recibe ampicilina y gentamicina }\end{array}$ & Fallece \\
\hline 9 & 2003 & $<1$ día & Sepsis & $\begin{array}{l}\text { Cesárea: } 30 \text { sem. PN } 1.665 \mathrm{~g} \text {. Apgar 3/8. No ATB materno previo. Aislamiento en sangre: S. pneumoniae } \\
+H \text {. influenzae no tipificable, } \beta \text { lactamasa negativa }\end{array}$ & Alta \\
\hline 10 & 2003 & 18 días & Sepsis & S. pneumoniae aislado en sangre, sin datos clínicos & \\
\hline 11 & 2004 & 2 días & MBA & $\begin{array}{l}\text { Parto: } 39 \text { sem. PN } 3.700 \mathrm{~g} \text { Apgar 9/10. REM } 6 \text { h. No ATB materno. A las } 48 \mathrm{~h} \text { fiebre }+ \text { SDR. S. pneumo- } \\
\text { niae aislado en LCR. Recibe ampicilina y gentamicina }\end{array}$ & Fallece \\
\hline 12 & 2004 & 27 días & Bacteriemia & $\begin{array}{l}\text { Parto: } 38 \text { sem. PN } 3.245 \text { g Apgar 9/10. REM } 1 \text { hora. Madre ingresa por sepsis puerperal al mes de vida } \\
\text { del RN, se aisla S. pneumoniae en sangre del RN }\end{array}$ & Alta \\
\hline 13 & 2005 & $<1$ día & Sepsis & $\begin{array}{l}\text { Parto: } 29 \text { sem. PN } 1.230 \mathrm{~g} \text { Apar 7/8. RAM intraparto. No ATB previo a parto. SDR e insuficiencia cardíaca } \\
\text { a la hora de vida. Opacidad bilateral en Rx Tx. Madre con sepsis luética. VDRL en sangre y LCR de RN } \\
\text { negativo. S. pneumoniae aislado en sangre. Recibe tratamiento con ampicilina y gentamicina }\end{array}$ & Alta \\
\hline 14 & 2005 & 18 días & MBA & S. pneumoniae aislado en LCR y sangre & Alta \\
\hline 15 & 2006 & 26 días & MBA & Parto: 39 sem. PN 3.155 g Apgar 9/10. S. pneumoniae aislado en LCR & Alta \\
\hline 16 & 2006 & $<1$ día & Sepsis & $\begin{array}{l}\text { Parto: } 39 \text { sem. PN } 2.900 \mathrm{~g} \text { Apgar 9/10. REM } 1 \mathrm{~h} \text {. No ATB previo a parto. Rx Tx normal. Madre con ITU a } \\
\text { E coli. S. pneumoniae aislado en sangre. Recibe tratamiento con ampicilina y gentamicina }\end{array}$ & Alta \\
\hline 17 & 2006 & 3 días & MBA & $\begin{array}{l}\text { Parto: } 38 \text { sem. PN } 2.630 \text { g Apgar 9/10. REM } 1 \text { hora. No ATB previo a parto. A los } 3 \text { días rechazo del } \\
\text { alimento e irritabilidad. Aislamiento de S. pneumoniae de sangre y LCR. Recibe tratamiento con ampi- } \\
\text { cilina y gentamicina }\end{array}$ & $\begin{array}{c}\text { Alta } \\
\text { Agravio neurológico }\end{array}$ \\
\hline 18 & 2006 & 14 días & MBA & S. pneumoniae aislado en LCR y sangre & Alta \\
\hline 19 & 2007 & $6 \mathrm{~h}$ & Neumonia & $\begin{array}{l}\text { Parto: } 37 \mathrm{sem} \text {. PN } 2.770 \mathrm{~g} \text { Apgar 9/10. REM } 7 \mathrm{~h} \text {. Sí ATB previo a parto. A las } 6 \mathrm{~h} \text { fiebre y SDR. Rx Tx } \\
\text { opacidad derecha. S. pneumoniae aislado en sangre. Recibe tratamiento con ampicilina y gentamicina }\end{array}$ & Alta \\
\hline 20 & 2008 & 1 día & MBA & $\begin{array}{l}\text { Cesárea: } 40 \text { sem. SFA. PN } 3.550 \text { g Apgar 3/6. REM intra cesárea. LAM. No ATB materno. Al nacer hepato- } \\
\text { esplenomegalia, ascitis, convulsiones. S. pneumoniae aislado en LCR y sangre }\end{array}$ & $\begin{array}{l}\text { Alta. Secuela: hidroce- } \\
\text { falia c/DVP, hipoacusia }\end{array}$ \\
\hline 21 & 2008 & 13 días & Sepsis & $\begin{array}{l}\text { Parto: } 40 \text { sem. PN } 3.300 \mathrm{~g} \text { Apgar 9/10. No ATB materno previo a parto. A los } 13 \text { días SDR y apneas. S. } \\
\text { pneumoniae aislado en sangre. Recibe tratamiento con ampicilina y cefotaxima }\end{array}$ & Alta \\
\hline 22 & 2008 & $<1$ día & Sepsis & $\begin{array}{l}\text { Parto: } 38 \text { sem. PN } 2.200 \mathrm{~g} \text { Apgar 9/10. No ATB previo al parto. SDR y apneas. S. pneumoniae aislado en } \\
\text { sangre. Recibe tratamiento con ampicilina y gentamicina }\end{array}$ & Fallece \\
\hline 23 & 2008 & 18 días & $\begin{array}{l}\text { Empiema/ } \\
\text { sepsis }\end{array}$ & $\begin{array}{l}\text { Cesárea: } 37 \mathrm{sem} \text {. PN } 2.100 \mathrm{~g} \text { Apgar 8/10. REM intra cesárea. No ATB materno. A los } 18 \text { días instala } \\
\text { fiebre, polipnea y rechazo del alimento. Rx Tx: consolidación parenquimatosa derecha con efusión pleu- } \\
\text { ral. S. pneumoniae aislado en sangre y líquido pleural. Recibe tratamiento con ampicilina y cefotaxima. }\end{array}$ & Fallece \\
\hline 24 & 2009 & $<1$ día & Sepsis & $\begin{array}{l}\text { Cesárea: } 35 \text { sem. PN } 2.665 \mathrm{~g} \text { Apgar } 6 / 8 \text {. REM } 5 \text { hs. Sí ATB materno. Al nacer SDR, polipnea. } \\
\text { S. pneumoniae aislado en sangre. Recibe tratamiento con cefotaxima }\end{array}$ & Alta \\
\hline 25 & 2010 & < 1 día & Sepsis & $\begin{array}{l}\text { Parto: } 39 \text { sem. PN } 3.090 \mathrm{~g} \text { Apgar 4/8. REM } 1 \text { hora. No ATB previo al parto. Fiebre + SDR + hiporreac- } \\
\text { tividad. Ingresa a UCl. AVM } 3 \text { días. S. pneumoniae aislado en sangre }\end{array}$ & Alta \\
\hline
\end{tabular}




\section{Antecedentes maternos}

Dos madres presentaron manifestaciones de infección al momento del parto. Una de ellas presentó una sepsis luética (caso 13) y otra estaba cursando una infección urinaria causada por Escherichia coli (caso 16). Otras dos reingresaron por sepsis (casos 7 y 12); no se logró obtener identificación del agente en ellas.

\section{Evolución y secuelas}

En cinco de los nueve casos de meningitis, se aisló $S$. pneumoniae en el LCR y en sangre. Tres de estos cinco RN presentaron importantes secuelas neurológicas. Ninguno de los cinco falleció.

\section{Letalidad}

En 20 casos se logró obtener el dato: fallecieron 6 (30\%): 3 meningitis (casos 1, 2 y 11), 2 sepsis (caso 8 y 22) y 1 empiema pleural (caso 23). Los casos 1 y 2 se presentaron en el año 2001, se trató de niños de 28 y 24 días respectivamente al momento del diagnóstico y en ambos casos se aisló $S$. pneumoniae serotipo 5 del LCR. El caso 11, se presentó en el año 2004; correspondió a un RN de 39 semanas, nacido por parto vaginal, REM de $6 \mathrm{~h}$, con un peso adecuado, vigoroso, que presentara las 48 h de vida fiebre y SDR. Se aisló $S$. pneumoniae, serotipo 5 en el LCR.

El caso 8, se presentó en el año 2002, se trató de un $\mathrm{RN}$ con 40 semanas de gestación y un peso adecuado a su edad, con rotura de membranas de 12 h, depresión neonatal moderada al minuto y vigoroso a los cinco minutos. Presentó un SDR, se aisló S. pneumoniae en sangre, serotipo $11 \mathrm{~A}$.

Los casos 22 (sepsis) y 23 (empiema pleural), se presentaron en el año 2008; el primero nació por parto vaginal a las 38 semanas, fue PEG (PN $2.200 \mathrm{~g}$ ), vigoroso. A las 24 h de vida manifiesta SDR y apneas. Se aisló $S$. pneumoniae en la sangre, no realizándose la serotipificación.

En el caso 23, se realizó cesárea a las 37 semanas (rotura de membranas intracesárea), peso al nacer 2.100 g. Vigoroso. A los 18 días presentó fiebre, polipnea y rechazo del alimento. La radiografía de tórax evidenció consolidación parenquimatosa en el lóbulo superior de pulmón derecho con efusión pleural. Se aisló $S$. pneumoniae no serotipificado en la sangre y el líquido pleural.

Las seis cepas de $S$. pneumoniae identificadas en los casos fatales eran sensibles a penicilina y todos los RN recibieron tratamiento antimicrobiano adecuado.

\section{Microbiología}

La Tabla 1 muestra los serotipos de $S$. pneumoniae y la susceptibilidad antimicrobiana. (ver nota al pie de la Tabla).

El serotipo 5 fue el más comúnmente aislado como causante de ENI en esta serie, identificándose en nueve $\mathrm{RN}$, siendo el último aislamiento de este serotipo en RN en el año 2008.

Los serotipos aislados según años, se muestran en la Tabla 3.

\begin{tabular}{|c|c|c|c|c|c|c|c|c|c|c|}
\hline & \multicolumn{2}{|c|}{ Serotipos vacunales VCN7 } & \multicolumn{4}{|c|}{ Serotipos vacunales adicionales en VCN13 } & \multicolumn{4}{|c|}{ Serotipos no vacunales } \\
\hline & $18 \mathrm{C}$ & $19 \mathrm{~F}$ & 1 & 5 & $7 F$ & $19 A$ & 8 & $11^{a}$ & Pool D & Sin serotipificar \\
\hline 2001 & & & $\mathrm{x}$ & $x x x x x$ & & $\mathrm{x}$ & & & & \\
\hline 2002 & & & & & & & & $\mathrm{x}$ & & \\
\hline 2003 & $x$ & & & & & & & & $x$ & \\
\hline 2004 & & & $x$ & $\mathrm{x}$ & & & & & & \\
\hline 2005 & & & $x$ & & $x$ & & & & & \\
\hline 2006 & $x$ & & $x$ & $x x$ & & & & & & \\
\hline 2007 & & $x$ & & & & & & & & \\
\hline 2008 & & & & $x$ & & & $x$ & & & $x x$ \\
\hline 2009 & & & & & & & & & & $x$ \\
\hline 2010 & & & $x$ & & & & & & & \\
\hline \multicolumn{11}{|l|}{2011} \\
\hline \multicolumn{11}{|l|}{2012} \\
\hline \multicolumn{11}{|l|}{2013} \\
\hline Total & 2 & 1 & 5 & 9 & 1 & 1 & 1 & 1 & 1 & 3 \\
\hline
\end{tabular}


Los 19 aislados de $S$. pneumoniae en RN los años 2001 a 2007, correspondieron a los serotipos 5 (n: 8), 1 (n: 4), 18C (n: 2), 7F (n: 1) 11A (n: 1), 19A (n: 1), 19F (n: 1) Pool D (n: 1). En el 2008 hubo cuatro RN con infección por $S$. pneumoniae, de los cuales se serotipificaron dos: 5 y 8 . En el período 2009-2013 se aislaron dos S. pneumoniae, siendo serotipificado uno de ellos que correspondió al serotipo 1 (incluído en la VCN 13 valente).

\section{Susceptibilidad antimicrobiana}

Veinticuatro de los 25 aislamientos fueron susceptibles a penicilinas y cefalosporinas de tercera generación. En el año 2009 se aisló de sangre una cepa de $S$. pneumoniae con una $\mathrm{CIM} \geq 2$ para penicilina. No se pudo identificar el serotipo. Se trató de un RN de 35 semanas, con una depresión neonatal moderada al minuto de nacer, que presentó un SDR. Recibió tratamiento con ampicilina y cefotaxima con excelente evolución.

\section{Discusión}

Esta es la primera investigación en Uruguay que incluye la descripción de las características clínicas y microbiológicas de la ENI en RN. Previamente en Uruguay se publicaron datos sobre ENI en niños bajo tres meses de edad ${ }^{19}$.

Si bien $S$. pneumoniae no es uno de los microorganismos más frecuentemente causante de infecciones en este grupo etario, se lo debe considerar como posible agente etiológico ${ }^{3,4,6,7}$.

Streptococcus pneumoniae no forma parte de la microbiota vaginal ${ }^{3,29}$; se estima que la incidencia de colonización en gestantes es inferior a $1 \%{ }^{4,6,30}$. La portación vaginal de este agente es un factor de riesgo en la transmisión de la infección al RN, así como de infección puerperal con morbi-mortalidad materna y del $\mathrm{RN}^{4,6,27,31,32}$. Se considera que la colonización materna por este agente, encierra un riesgo mayor de infección en el $\mathrm{RN}$ que en el caso de colonización materna por Streptococcus agalactiae ${ }^{2}$. Se ha sugerido que el hallazgo de este agente en un exudado vaginal en la mujer embarazada debe considerarse patológico y se debe plantear la realización de tratamiento antimicrobiano durante el parto, con control estricto posterior del $\mathrm{RN}$ y eventual tratamiento antimicrobiano a éste $3,4,6,7,30,31,33-35$.

La vía de transmisión hacia el RN puede ser vertical; por vía transplacentaria en caso de bacteriemia materna o durante el nacimiento a partir de la colonización vaginal materna $^{3,4,7,32}$. La infección por vía transplacentaria puede determinar infección fetal y parto prematuro ${ }^{2,4}$; sumando a las complicaciones de la infección, las de la prematurez. En este grupo de niños, sólo tres de los 16 RN en los que se obtuvo el dato fueron $\mathrm{RN}$ de pretérmino $(29,30$ y 35 semanas).
Otra vía de adquisición de la infección es la respiratoria, a partir de un portador faríngeo, ya fuese su madre, otros niños o adultos cercanos al $\mathrm{RN}^{4}$. En uno de los casos de esta serie (caso 12) fue la madre quien ingresó a los 27 días del parto con sepsis puerperal. Se realizó hemocultivo al RN asintomático, aislándose $S$. pneumoniae, serotipo 1 .

Como la transmisión vertical no es la única forma de infección del RN, adquiere especial importancia el desarrollo de otras estrategias que permitan disminuir el riesgo de infecciones neumocóccicas en el RN.

Los RN con infecciones neumocóccicas se presentan con síntomas indistinguibles a los de infecciones neonatales por otros agentes. Más de la mitad de los RN de esta serie presentaron síntomas en sus primeras $72 \mathrm{~h}$ de vida, similar a otros estudios ${ }^{2}$ lo que sugiere fuertemente en éstos, la adquisición de $S$. pneumoniae a partir del tracto genital materno ${ }^{36}$.

Estudios previos asociaron las infecciones neumocóccicas neonatales con rotura prematura de membranas, prematurez y bajo peso al nacer ${ }^{7,34}$, pero posteriormente, un estudio en el cual analizaron 119 casos de infecciones neumocóccicas neonatales no encontró esa asociación ${ }^{37}$.

Otro estudio sobre ENI en lactantes bajo 90 días realizado en el Reino Unido, no demostró asociación con prematurez o existencia de co-morbilidades y mortalidad ${ }^{2}$. En la presente investigación, los RN con factores de riesgo como nacimiento pretérmino y depresión neonatal presentaron una buena evolución. No hubo casos de rotura de membranas de más de $12 \mathrm{~h}$. Los dos RN que presentaron un peso PEG (ambos de término) fallecieron.

En cuanto a las localizaciones de la infección, la sepsis y meningitis fueron las más frecuentes, no identificándose en esta serie, infecciones osteo-articulares como en otras publicaciones $^{2,3}$.

En esta investigación más de $50 \%$ de los aislados correspondieron a los serotipos 5 y 1 . La mayoría de los casos ocurrió antes de la implementación de la vacunación universal con VCN 13.

Los serotipos 5 y 1 fueron los únicos causantes de meningitis en esta serie; no identificándose casos de meningitis por serotipo $7 \mathrm{~F}$, el cual fue el de mayor frecuencia en meningitis y otras infecciones neumocóccicas en lactantes bajo 90 días de vida en un estudio realizado en Utah, E.U.A. ${ }^{36}$ y en lactantes bajo 60 días de vida en Alemania, previo a la implementación de VPN 7 valente ${ }^{38,39}$.

Es destacable que de todos los pacientes que presentaron secuelas (tres de los nueve pacientes con meningitis) y en tres de los seis fallecidos, $S$. pneumoniae fue aislado en sangre, además de LCR en los casos de meningitis y líquido pleural en el caso de empiema pleural. En un caso, el hallazgo de $S$. pneumoniae en sangre no determinó síntomas en el RN.

La letalidad observada, de $30 \%$ en este grupo, es bastante mayor a la presentada en una serie británica ${ }^{2}$ y 
mucho menor a la reportada por otros estudios, la cual es cercana a $50 \%{ }^{4,37}$. No fallecieron RN por ENI en el período post-vacunación.

El 15,8\% de los aislados identificados entre los años 2001 y 2007 corresponden a serotipos incluidos en la VCN7 y $89,4 \%$ en la VCN13, años en que aún no se administraban estas vacunas en Uruguay.

En nuestro país, en el período pre-vacunal, los serotipos más frecuentes aislados en los niños bajo dos años fueron los serotipos 14, 6B, 5 y 3; mientras que entre los dos y los 15 años, fueron en orden decreciente de frecuencia: 14, 1, 5 y 3. Los serotipos más frecuentemente aislados en población mayor de 15 años en Uruguay en el período que comprende este estudio fueron el $1,3,7 \mathrm{~F}$ y $5^{40}$.

En los RN, el serotipo 5 fue el más frecuentemente aislado; no se identificó a partir del año 2008 (año en que se inició la vacunación con VCN7). En el año 2010 se identificó el último aislamiento de $S$. pneumoniae en $\mathrm{RN}$, siendo éste del serotipo 1 (incluido en la VCN13). A diferencia con otros estudios ${ }^{10,36}$ no se produjo un aumento de la identificación de $S$. pneumoniae no vacunales.

Si bien los serotipos 1 y 5 presentan oscilaciones periódicas en su incidencia ${ }^{41,42}$, la disminución en la circulación de $S$. pneumoniae, originada por la vacunación de los niños bajo un año, podría ser uno de los factores que ha contribuído en la disminución de estas infecciones neonatales. El 86\% de las cepas de $S$. pneumoniae identificados en esta serie se encuentra incluído en la VCN administrada en Uruguay.

En cuanto a la susceptibilidad antimicrobiana, sólo se aisló una cepa de $S$. pneumoniae resistente a penicilina, pero esto no determinó que el $\mathrm{RN}$ presentara una mala evolución.

Conclusión: Se produjo una disminución de los casos de ENI neonatal en el período post-vacunación. Esta disminución podría atribuirse, en parte, a la disminución de la circulación de $S$. pneumoniae por el efecto rebaño, luego de la vacunación universal. Es imprescindible continuar con la vigilancia epidemiológica de estas infecciones, con el fin de evaluar el real impacto de la vacunación universal.

Agradecimientos: a Adriana Alfonso por los datos aportados.

\section{Resumen}

Introducción: Streptococcus pneumoniae infrecuentemente produce infecciones en recién nacidos $(\mathrm{RN})$, presentando elevada morbi-mortalidad. En Uruguay, en 2008 se incorporó al calendario de inmunizaciones infantil la vacuna conjugada neumocóccica (VCN) 7 valente, (sustituída por VCN13 en 2010). La vacunación comienza a los dos meses de vida. Se espera que la vacunación universal tenga impacto en la protección de RN. Objetivo: Describir la presentación clínica, microbiología y evolución de RN con enfermedad neumocóccica invasora (ENI), identificados en dos hospitales de Uruguay, años 2001-2007 (pre-vacunación), 2008 (intervención) y 2009-2013 (postvacunación). Material y Métodos: Estudio descriptivo, retrospectivo. Lugar: Hospital Pereira Rossell y Hospital Paysandú. Se incluyeron todos los aislados de S. pneumoniae a partir de líquidos normalmente estériles. Fuente de datos: laboratorios de bacteriología e historias clínicas. Análisis estadístico: frecuencias absolutas, relativas, tasas y riesgo relativo. Resultados: RN con ENI: 25, sepsis (n: 13), meningitis (n: 9), bacteriemia (n: 1), neumonía con empiema (n: 1), neumonía (n: 1). Incidencia de ENI en el período pre-vacunación 19/25, tasa 0,30/1.000 nacimientos; tasa post-vacunación: 0,04/1.000. Riesgo relativo 5,9. Fallecimientos: 6/20 (30\%): meningitis (n: 3), sepsis (n: 2), empiema (n: 1). Los serotipos más frecuentes fueron: 5 y 1 (14/25). Susceptibles a penicilina: 24/25. Discusión: Los síntomas fueron indistinguibles de infecciones causadas por otros patógenos. Disminuyeron los casos de ENI y no ocurrieron fallecimientos en el período post-vacunación. No aumentaron los serotipos no vacunales.

\section{Referencias bibliográficas}

1.- O'Brien K L, Wolfson L J, Watt J P, Henle E, Deloria-Knoll M, Mc Call N, et al. Hib and Pneumococcal Global Burden of Disease Study Team. Burden of disease caused by Streptococcus pneumoniae in children younger than 5 years: global estimates. Lancet 2009; 374 (9693): 893-902.

2.- Ladhani S N, Andrews N J, Waight P, Borrow R, Slack M P, Miller E. Impact of the 7-valent pneumococcal conjugate vaccine on invasive pneumococcal disease in infants younger than 90 days in England and Wales. Clin Infect Dis 2013; 56 (5): 633-40.

3.- Hoffman J A, Mason E O, Schutze G E, Tan T
Q, Barson W J, Givner L B, et al. Streptococcus pneumoniae infections in the neonate. Pediatrics. 2003; 112: 1095-102.

4.- Singh J, Dick J, Santosham M. Colonization of the female urogenital tract with Streptococcus pneumoniae and implications for neonatal disease. Pediatr Infect Dis J 2000; 19 (3): 260-2.

5.- Poehling K A, Talbot T R, Griffin M R, Craigg A S, Whitney C G, Zell E, et al. Invasive pneumococcal disease among infants before and after introduction of pneumococcal conjugate vaccine. JAMA 2006; 295 (14): 1668-74.

6.- Marcus KA, Verduin CM. Invasieve pneumokokken infectie bij 5 pasgeborenen, 1996-2004. Ned Tijdschr Geneeskd 2007; 151: 2527-30.
7.- $\quad$ Simpson J M, Patel J S, Ispahani P. Streptococcus pneumonia invasive disease in the neonatal period: an increasing problem? Eur J Pediatr 1995; 154: 563-6.

8.- Kaplan S L, Mason E O Jr, Wald E R, Schutze G E, Bradley J S, Tan T Q, et al. Decrease of invasive pneumococcal infections in children among 8 children's hospitals in the United States after the introduction of the 7-valent pneumococcal conjugate vaccine. Pediatrics 2004; 113 (3 pt 1): 443-9.

9.- Whitney C G, Farley M M, Hadler J, Harrison L H, Bennett N M, Lynfield R, et al. Active Bacterial Core Surveillance of the Emerging Infections Program Network. Decline in invasive pneumococcal disease after the 
introduction of protein-polysaccharide conjugate vaccine. N Engl J Med 2003; 348 (18): 1737-46.

10.- Pilishvili T, Lexau C, Farley M M, Hadler J, Harrison L H, Bennett N M, et al. Active Bacterial Core Surveillance/Emerging Infections Program Network. Sustained reductions in invasive pneumococcal disease in the era of conjugate vaccine. J Infect Dis 2010; 201 (1): 32-41.

11.- Miller E, Andrews N J, Waight P A, Slack M P, George R C. Herd immunity and serotype replacement 4 years after seven-valent pneumococcal conjugate vaccination in England and Wales: an observational cohort study. Lancet Infect Dis 2011; 11: 760-8.

12.- Black S B, Shinefield H R, Ling S, Hansen J, Fireman B, Spring D, et al. Effectiveness of heptavalent pneumococcal conjugate vaccine in children younger than five years of age for prevention of pneumonia. Northern California Kaiser Permanente Vaccine Study Center Group. Pediatr Infect Dis J 2002; 21: 810-5.

13.- Lucero M G, Nohynek H, Williams G, Tallo V, Simões E A, Lupisan S, et al. Efficacy of an 11-valent pneumococcal conjugate vaccine against radiologically confirmed pneumonia among children less than 2 years of age in the Philippines: a randomized, double-blind, placebo-controlled trial. Pediatr Infect Dis J 2009; 28: 455-62.

14.- Grijalva C G, Nuorti J P, Arbogast P G, Martin S W, Edwards K M, Griffin M R. Decline in pneumonia admissions after routine childhood immunization with pneumococcal conjugate vaccine in the USA: a time-series analysis. Lancet 2007; 369: 1179-86.

15.- Lexau C A, Lynfield R, Danila R, Pilishvili T, Facklam R, Farley M M, et al. Changing epidemiology of invasive pneumococcal disease among older adults in the era of pediatric pneumococcal conjugate vaccine. JAMA 2005; 294: 2043-51.

16.- Millar E, Watt J, Bronsdon M, Dallas J, Reid R, Santosham M, et al. Indirect effect of 7 -valent pneumococcal conjugate vaccine on pneumococcal colonization among unvaccinated household members. Clin Infect Dis 2008; 47: 989-96.

17.- Pírez M C, Algorta C, Cedres A, Sobrero H, Varela A, Giachetto $\mathrm{G}$ et al. Impact of universal pneumococcal vaccination on hospitalizations for pneumonia and meningitis in children in Montevideo, Uruguay. Pediatr Infect Dis J 2011; 30 (8): 669-74.

18.- Dagan R, Melamed R, Muallem M, Piglansky L, Greenberg D, Abramson O, et al. Reduction of nasopharyngeal carriage of pneumococci during the second year of life by a heptavalent conjugate pneumococcal vaccine. $\mathrm{J}$ Infect Dis 1996; 174: 1271-8.
19.- Pujadas M, Díaz Roselló J. Infecciones por Streptococcus pneumoniae en los primeros meses de vida: diagnóstico y prevención. En: Hortal M, Iraola I, Camou T editores. Avances multidisciplinarios para el control integral de S. pneumoniae: 10 años de experiencia. $1^{\circ}$ ed. Montevideo: OPS; 2004. Pág 119-24.

20.- Uruguay, Ministerio de Salud Pública. Dirección General de Salud. División Epidemiología. Certificado esquema de vacunación año 2008. Obtenido de: www.msp.gub.uy [consulta: diciembre de 2013].

21.- Uruguay, Ministerio de Salud Pública. Dirección General de Salud. División Epidemiología. Certificado esquema de vacunación año 2010. Obtenido de: www.msp.gub.uy [consulta: diciembre de 2013].

22.- Uruguay, Ministerio de Salud Pública. Dirección General de Salud. División Epidemiología. Campaña de nivelación con vacuna conjugada 13-valente. Obtenido de: www.msp.gub.uy [consulta: diciembre de 2013].

23.- Uruguay, Programa nacional operativo de inmunizaciones. [Comisión horaria de la lucha antituberculosas y enfermedades prevalentes. web site]. Obtenido de: http://www.chlaep.org. uy/programas-inmunizaciones-estadisticas.php [consulta: diciembre de 2013]

24.- Hortal M, Estevan M, Laurani H, Iraola I, Meny M. Hospitalized children with pneumonia in Uruguay: Pre and post introduction of 7 and 13-valent pneumococcal conjugated vaccines into the National Immunization Program. Vaccine 2012; 30 (33): 4897-5058.

25.- Uruguay, Ministerio de Salud Pública. Dirección General de Salud. División Epidemiología. Estadísticas de Natalidad. Obtenido de: www. msp.gub.uy [consulta: noviembre de 2014].

26.- Uruguay, Instituto Nacional de Estadística. Censo Poblacional 2011. Obtenido de: www. ine.gub.uy/censos2011/index.html [consulta: noviembre de 2014].

27.- Versalovic J, Carroll K C, Funke G, Jorgensen J H, Landry M L, Warnock D W. Manual of Clinical Microbiology. 10th ed. Washington DC: American Society for Microbiology; 2011.

28.- Institute Antimicrobial Susceptibility Testing Standards (CLSI). Performance Standards for Antimicrobial Susceptibility Testing; Twentysecond Informational Supplement, CLSI document M100-S22. Wayne, PA: Clinical and Laboratory Standards Institute; 2012.

29.- Nader R, Tondeur S, Barrans A, Gevaudan A, Lamy B. Infection néonatale précoce à Streptococcus pneumoniae. Ann Biol Clin 2005; 63 (6): 643-6.

30.- Sallam A, Paes B. Streptococcus pneumoniae an old bug with significant maternal-newborn implications. Am J Perinatol 2004; 21: 491-5.
31.- de Alba C, Ramos J T, Gómez del Castillo E. El neumococo: ¿un germen nuevo en el recién nacido? An Esp Pediatr 2001; 54 (4): 390-3.

32.- Hughes B R, Mercer J L, Gosbel L B. Neonatal pneumococcal sepsis in association with fatal maternal pneumococcal sepsis. Aust N Z J Obstet Gynaecol 2001; 41: 457-8.

33.- Primhak R A, Tanner M S, Spencer R C. Pneumococcal infection in the newborn. Arch Dis Child 1993; 69: 317-8.

34.- Geelen S P M, Gerards L J, Fleer A. Pneumococcal septicemia in the newborn. A report on seven cases and a review of the literature. J Perinat Med 1990; 18: 125-9.

35.- Karabayir N, Hatipoglu N, Adal E, Sanli K. A rare case of sepsis in newborn: Streptococcus pneumoniae septicemia. Arch Gynecol Obstet. 2010; 282 (5): 591-2.

36.- Olarte L, Ampofo K, Stockmann K, Mason E, Daly J, Pavia A, et al. Invasive pneumococcal disease in infants younger than 90 days before and after introduction of PCV7. Pediatrics. 2013; 132: e17-e24.

37.- Gómez M, Alter S, Kumar M L, Murphy S, Rathore M H. Neonatal Streptococcus pneumoniae infection: case reports and review of the literature. Pediatr Infect Dis J 1999; 18: 1014-8.

38.- Imöhl M, van der Linden M, Mutscher C, Reinert RR. Serotype distribution of invasive pneumococcal disease during the first 60 days of life. Vaccine 2010; 28 (30): 4758-62.

39.- Imöhl M, Reinert R R, Ocklenburg C, van der Linden M. Association of serotypes of Streptococcus pneumoniae with age in invasive pneumococcal disease. J Clin Microbiol 2010; 48 (4): 1291-6.

40.- Organización Panamericana de la Salud. Vigilancia Sanitaria, Prevencion y Control de Enfermedades (HSD) Informes Regionales de SIREVA II. Washington, DC: OPS; 2011. 20002005; 2006; 2007; 2008; 2009; 2010; 2011; 2012: (Serie Documentos técnicos. Tecnologías esenciales de salud. THS/EV). Washington: OPS; 2008- 2013. Obtenido de: http://www. paho.org/hq/index.php?option=com_content\& view $=$ category \&layout $=$ blog $\& i d=3609 \&$ Item id=3953 [consulta: diciembre de 2013].

41.- Dagan R, Gradstein S, Belmaker I, Porat N, Siton Y, Weber G, et al. An outbreak of Streptococcus pneumoniae serotype 1 in a closed community in Southern Israel. Clin Infect Dis 2000; 30: 319-21.

42.- Rommery M G, Hull M W, Gustafson R, Sandhu J, Champagne S, Wong T, et al. Large community outbreak of Streptococcus pneumoniae serotype 5 invasive infection in an impoverished urban population. Clin Infect Dis 2008; 47: 768-74. 